\title{
Temporal Fluctuations of Gut Microbiota and Bile Acid Metabolism in Critically III Children
}

\author{
Josef Wagner

\section{Anisha Wijeyesekera} \\ University of Reading \\ Marcus de Goffau \\ University of Cambridge

\section{Sarah Thurston} \\ University of Cambridge \\ John A Clark \\ University of Cambridge \\ Deborah K. White \\ University of Cambridge \\ Jenna Ridout \\ $\mathrm{EACH}$, Church Lane
}

lain Robert Louis Kean ( $\nabla$ irlk2@cam.ac.uk)

University of Cambridge https://orcid.org/0000-0003-1066-8285

The Peter Doherty Institute for Infection and Immunity

\section{Shruti Agrawal}

Cambridge University Hospitals NHS Foundation Trust

\section{Riaz Kayani}

Cambridge University Hospitals NHS Foundation Trust

\section{Roddy O'Donnell}

Cambridge University Hospitals NHS Foundation Trust

\section{Padmanabhan Ramnarayan}

Great Ormond Street Hospital for Children NHS Foundation Trust

\section{Mark J Peters}

UCL Great Ormond Street Institute of Child Health

Nigel Klein

UCL Great Ormond Street Institute of Child Health

\section{Elaine Holmes}

Imperial College London

Julian Parkhill

University of Cambridge

\section{Stephen Baker}


University of Cambridge

\section{Nazima Pathan}

University of Cambridge

\section{Short report}

Keywords: paediatric, microbiome, critical illness, bile acid

Posted Date: June 29th, 2021

DOI: https://doi.org/10.21203/rs.3.rs-647124/v1

License: (c) (1) This work is licensed under a Creative Commons Attribution 4.0 International License. Read Full License 


\section{Abstract}

Background: Critical illness frequently requires the use of broad-spectrum antimicrobials to treat lifethreatening infection. The resulting impact on microbiome diversity is profound, influencing gastrointestinal fermentation endpoints, host immune response and metabolic activity including the conversion of primary bile acids to secondary bile acids. We previously observed reduced fermentation capacity in the gut microbiota of critically ill children upon hospital admission, but the functional recovery trajectory of the paediatric gut microbiome during critical illness has not been well defined. Here, we longitudinally studied the intestinal microbiome and faecal bile acid profile of critically ill children during hospitalisation in a paediatric intensive care unit (PICU). The composition of the microbiome was determined by sequencing of the 16s rRNA gene, and bile acids were measured from faecal water by liquid chromatography hyphenated to mass spectrometry.

Results: In comparison to admission faecal samples, members of Clostridium cluster XIVa and Lachnospiraceae recovered during the late-acute phase (days 8-10) of hospitalisation. Patients with infections had a lower proportion of Lachnospiraceae in their gut microbiota than control microbiota and patients with admitting diagnoses. The proportion of Recovery Associated Bacteria (RAB) was observed to decline with the length of PICU admission. Additionally, the proportions of RAB were reduced in those with systemic infection, respiratory failure, and undergoing surgery. Notably, Clostridioides were positively associated with the secondary bile acid deoxycholic acid, which we hypothesised to driven by secondary bile acid induced sporulation; the ratio of primary to secondary bile acids demonstrated recovery during critical illness.

Conclusion: The recovery of secondary bile acids occurs quickly after intervention for critical illness. Bile acid recovery may be induced by the Lachnospiraceae, the composition of which shifts during critical illness. Our data suggest that gut health and early gut microbiota recovery can be assessed by readily quantifiable faecal bile acid profiles.

\section{Full Text}

This preprint is available for download as a PDF.

\section{Figures}



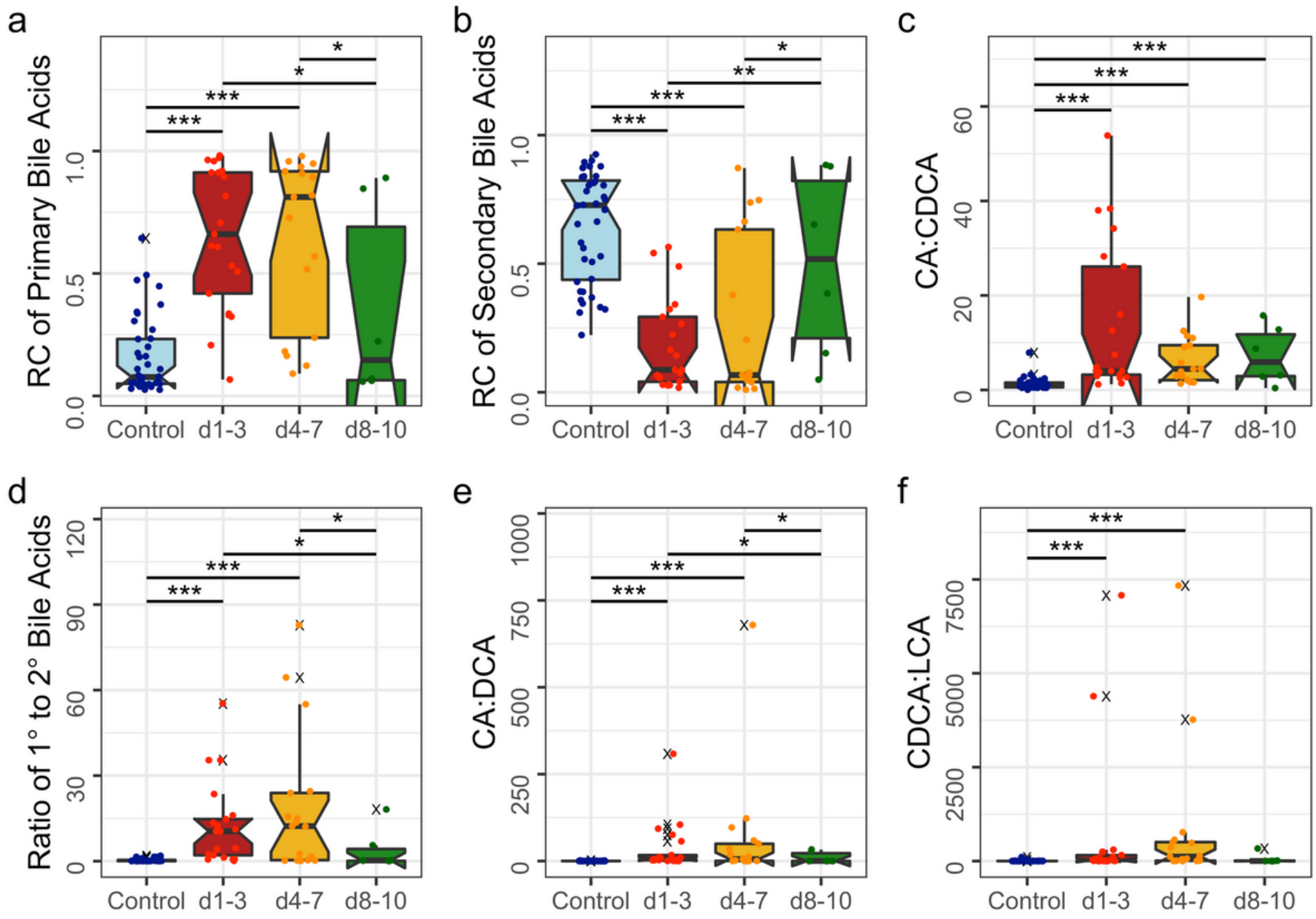

\section{Figure 1}

Primary and secondary bile acids a) primary bile acids (Cholic acid (CA), Chenodeoxycholic acid (CDCA), Glycochenodeoxycholic acid (GCDCA), and Taurocholic acid (TCA)) in faecal water measured by LC-MS as a ratio of total measured bile acid. b) secondary bile acids (Deoxycholic Acid (DCA), Lithocholic acid (LCA), Isolithocholic acid (ILCA), Ursodeoxycholic acid (UDCA), 3a-Hydroxy,12-Ketolithocholic acid (3a$\mathrm{H}, 12-\mathrm{KLCA})$, Taurohyocholic acid (THCA), and Glycoursodeoxycholic acid (GUDCA)) in faecal water measured by LC-MS as a ratio of total measured bile acid. A significant reduction to the relative concentration of total secondary bile acids versus the total measured bile acid pool was observed for patients sampled at day 1-3 and day 4-7 compared to the control. The median proportion of these secondary bile acids at days 8-10 was comparable to that of the aged-matched controls, but we observed a significant increase of patient bile acids at days 8-10 above the median for patients at day 1-3. c) the ratio of $C A$ to $C D C A$, two species of primary bile acids in the human gut. The control samples had a median CA:CDCA ratio of 0.9 ; the median CA:CDCA ratios on days 1-3, 4-7, an 8-10 in the faecal samples of the critically patients were $4.4,4.4$, and 5.9 , respectively. d) Ratio of primary bile acids to secondary bile acids. e) ratio of CA to DCA in faecal water.The ratio of CA to DCA was elevated in across all patient timepoints compared to the control. Patient samples collected on days 8-10 had lower ratios of CA:DCA 
than patients at day 1-3. f) ratio of CDCA to LCA and ILCA. CDCA:LCA ratios were increased compared to controls in patient samples from day 1-3 and day 4-7, returning to control levels at days 8-10. ConoverIman significance ${ }^{* *} p \leq 0.001, * * p \leq 0.01, * p \leq 0.05, p>0.05=$ not shown.
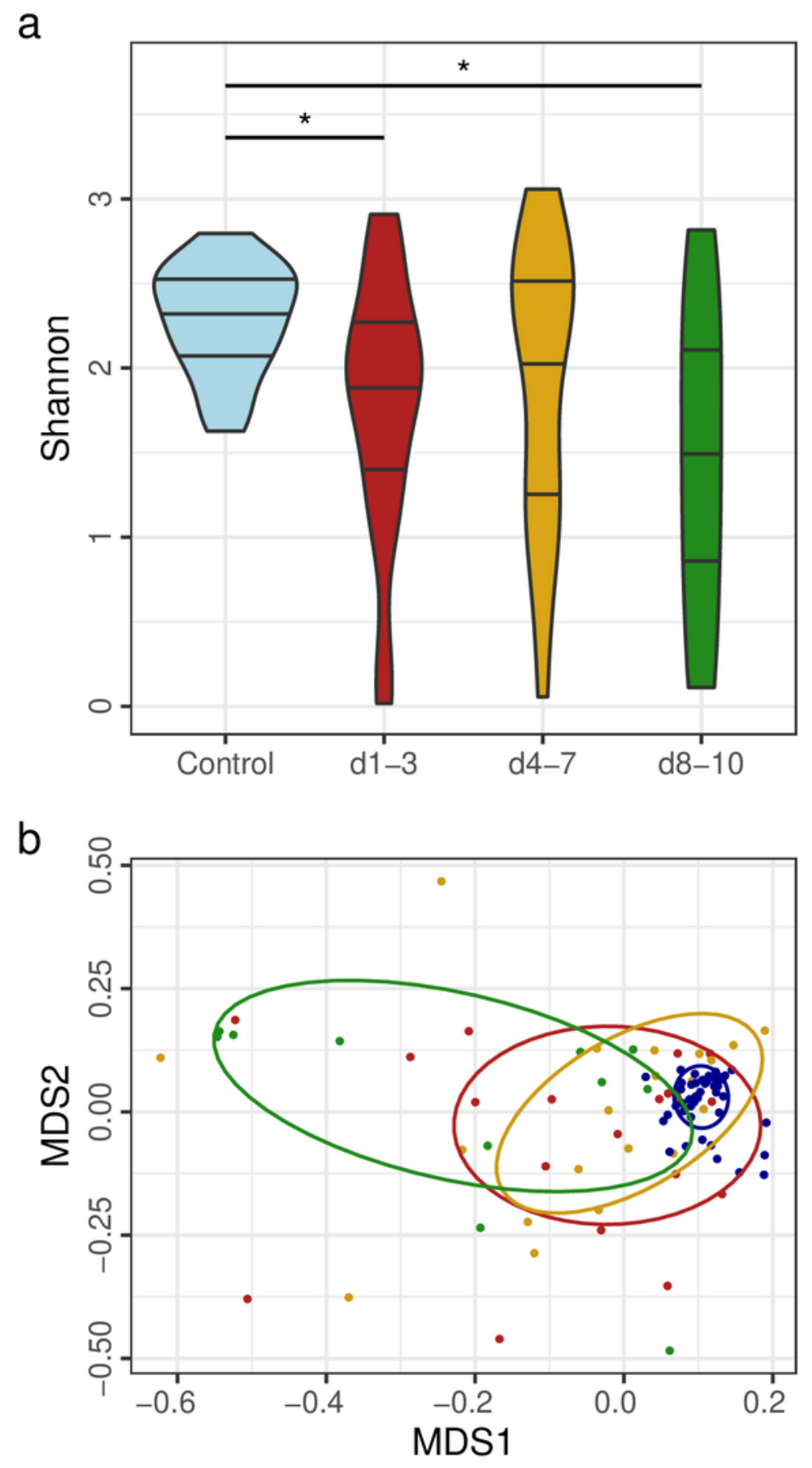

$\rightarrow$ Control $\rightarrow \mathrm{d} 1-3 \rightarrow \mathrm{d} 4-7 \rightarrow \mathrm{d} 8-10$

Figure 2

Bacterial diversity, and abundance of beneficial bacteria. a) Shannon diversity of sampling groups, bars indicate 1 st 2 nd and 3 rd quartiles. Due to the broad range of alpha-diversity at day 1-3, day 4-7 and days 
8-10, only the median alpha diversity of samples collected at days 8-10 was significantly lower than that of the control group after correcting for multiple comparisons. b) NMDS clustering of beta diversity of bacterial populations compared between sampling groups. PERMANOVA $p$-value $=0.001$. Ellipses indicate $60 \%$ similarity. Conover-Iman significance ${ }^{* *} p \leq 0.001, * * p \leq 0.01,{ }^{*} p \leq 0.05, p>0.05=$ not shown.

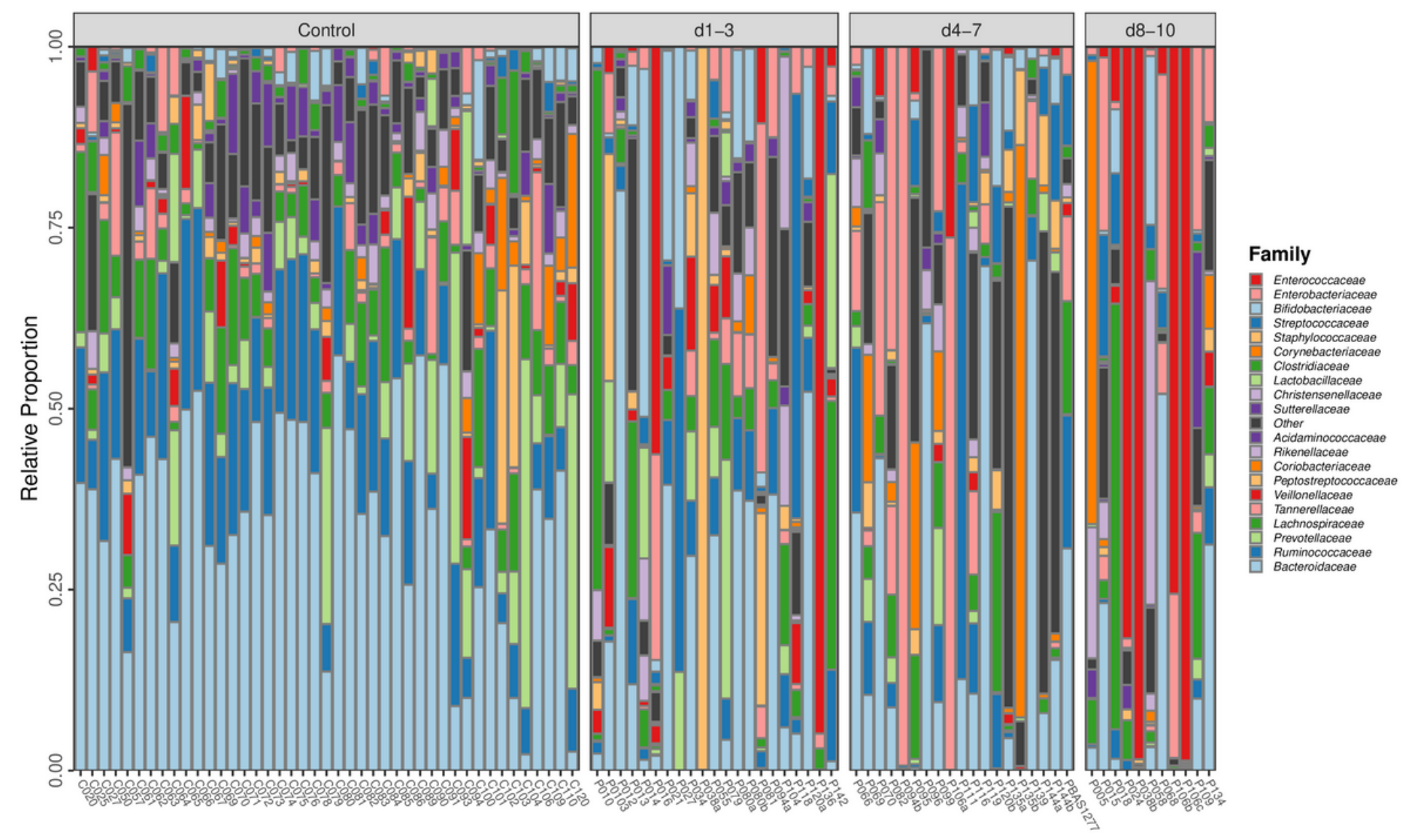

\section{Figure 3}

Relative proportion of bacteria identified by 16S rRNA gene sequencing. Bacterial 16S rRNA gene counts are represented as proportional abundance at Family level for each sample. The majority of analysed $16 \mathrm{~S}$ rRNA gene counts are within $12 \mathrm{k}$ to $16 \mathrm{k}$ reads. The ten most abundant families in the control microbiomes were the Bacteroidaceae, Ruminococcaceae, Prevotellaceae, Lachnospiraceae, Tannerellaceae, Veillonellacea, Peptostreptococcaceae, Coriobacteriaceae, Rikenellaceae and Acidaminococcaceae. These ten families represent a median of $82.8 \%$ (IQR 12.7\%) of the control faecal microbiota and a median of $45.3 \%$ (IQR $57 \%$ ) of the patient faecal microbiota. The ten most abundant bacterial families present in the patient faecal microbiome, which were not highly represented in the control samples were the Enterococcaceae, Enterobacteriacea, Bifidobacteriaceae, Streptococcaceae, Staphylococcaceae, Corynebacteriaceae, Clostridiaceae, Lactobacillaceae, Christensenellaceae and the Sutterellaceae. These families represented a median of 7.9\% (IQR $8.8 \%$ ) of the control microbiome, and a median of $31.1 \%$ (IQR $64.8 \%$ ) of the patient samples. 


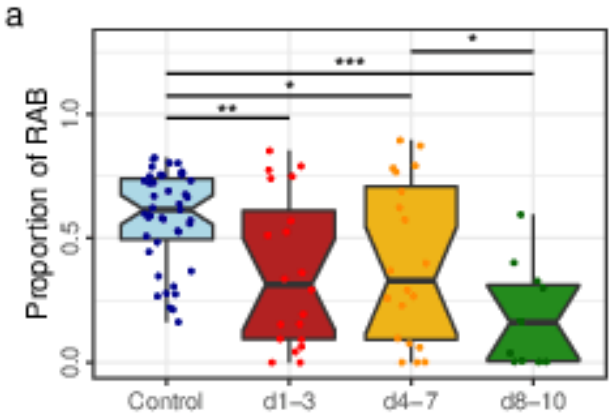

b

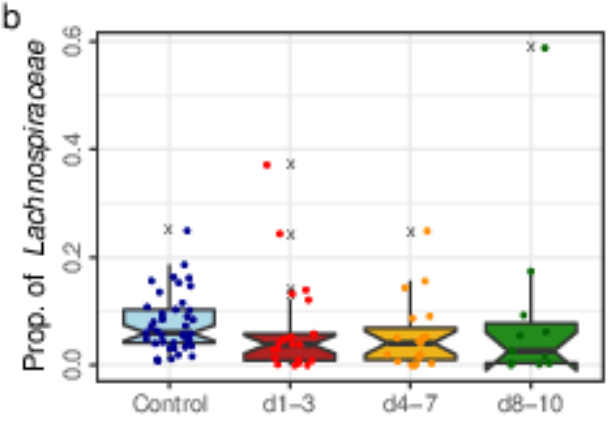

Figure 4

Relative abundance of recovery associated bacteria (RAB) and Lachnospiraceae. a) Proportion of RAB present in microbiomes. Patient samples from day 1-3, day 4-7 and days 8-10 were significantly reduced from the median level of the control group. RAB at days 8-10 were also significantly reduced from the median RAB level at day 4-7. b) Proportion of Lachnospiraceae present in microbiomes. No significant difference was observed between groups after controlling for multiple comparisons. Conover-Iman significance $* \star * p \leq 0.001,{ }^{* \star} p \leq 0.01, * p \leq 0.05, p>0.05=$ not shown.

\section{Supplementary Files}

This is a list of supplementary files associated with this preprint. Click to download.

- Kean2021FigureS1.eps

- Kean2021Figures2.eps

- Kean2021FigureS3.eps

- Kean2021FigureS4.eps

- Kean2021Rscripts.rmd

- Kean2021datatable.csv

- Kean2021familytaxonomy.csv

- Kean2021 supplementarymethods.docx

- Kean2021 supplementarytables.pdf 